\title{
An elderly couple with COVID-19 pneumonia treated in Singapore: contrasting clinical course and management
}

Singapore Med J 2020; 61(7): 392-394 https://doi.org/10.11622/smedj.2020064

Dear Sir,

Singapore reported its first case of coronavirus disease 2019 (COVID-19) infection on 23 January 2020. ${ }^{(1)}$ After the initial first wave of cases - most of whom had a history of travel from China, with limited local clusters ${ }^{(2)}$ - the second wave of the outbreak saw imported cases from Europe, the United States and the surrounding ASEAN (Association of Southeast Asian Nations) countries. ${ }^{(3)}$ There were also increasing numbers of unlinked cases, suggesting the occurrence of local community transmission. We herein describe the clinical illness of an elderly couple, foreign nationals who had travelled to Singapore on 9 March 2020 as part of a three-generation family group.

Patient A was a 75-year-old woman who arrived in Singapore on 9 March 2020 with her family. She developed a persistent dry cough on the evening of 8 March. On the morning of 10 March, she had a fever and attended the 24-hour clinic at Gleneagles Hospital. She had no gastrointestinal or genitourinary symptoms, and no contact with persons known to have COVID-19. She was on daily treatment for hypertension with nifedipine and irbesartan. There was a known allergy to sulfa drugs.

At midday of 10 March, her temperature was $38.4^{\circ} \mathrm{C}$ and blood pressure (BP) $143 / 67 \mathrm{mmHg}$. Examinations of the heart, lungs, abdomen and peripheral lymphatic system were normal. She was admitted to Gleneagles Hospital and placed on intravenous (IV) hydration and medications for symptom relief. Chest radiography on the same day did not demonstrate any mass, consolidation or pleural effusion. Investigations on 10 March showed the following: haemoglobin (Hb) $12.6 \mathrm{~g} / \mathrm{dL}$; white blood cells $(\mathrm{WBC}) 5.94 \times 10^{9} / \mathrm{L}$; platelet $255.0 \times 10^{9} / \mathrm{L}$; C-reactive protein $(\mathrm{CRP})<9.9 \mathrm{mg} / \mathrm{L}$; negative dengue nonstructural protein 1 (NS1) antigen; weakly positive dengue immunoglobulin G (IgG); and negative dengue immunoglobulin M (IgM). Nasopharyngeal swabs for severe acute respiratory syndrome coronavirus 2 (SARS-CoV-2) by polymerase chain reaction (PCR) test were positive on 10 and 11 March. Notification for COVID-19 was made to the Ministry of Health $(\mathrm{MOH})$ on the evening of 11 March, and a request for transfer of care to the National Centre for Infectious Diseases (NCID) was made.

Patient B was an 82-year-old man who developed a fever on the evening of 8 March 2020. There were no symptoms that were referable to the respiratory, gastrointestinal or genitourinary tracts. He saw a doctor in Indonesia on 9 March, but investigations, including full blood count, urine microscopy and dengue tests, were unremarkable. He arrived with his family for a holiday in Singapore on 9 March. The fever recurred, and he attended the 24-hour clinic at Gleneagles Hospital for evaluation. At midday on 10 March, he had no other symptoms other than fever. He had no contact with persons known to have COVID-19.

The patient was on treatment for hypertension (bisoprolol, nifedipine and valsartan), diabetes mellitus (linagliptin), dyslipidaemia (rosuvastatin) and gout (allopurinol), and was known to have moderate coronary artery disease and Stage 3 chronic kidney disease. He had a minor allergy to contrast. In July 2019, he had undergone ablation for atrial flutter. He had stopped smoking for more than 30 years.

At midday on 10 March, his temperature was $37.5^{\circ} \mathrm{C}$ (Tmax $38^{\circ} \mathrm{C}$ earlier) and BP $118 / 55 \mathrm{mmHg}$. Examinations of the heart, lungs, abdomen and peripheral lymphatic system were normal. He was admitted to Gleneagles Hospital and placed on IV hydration and medications for symptom relief. Chest radiography demonstrated cardiomegaly, but no pulmonary mass, consolidation or pleural effusion was seen. Investigations on 10 March showed the following: Hb $12.7 \mathrm{~g} / \mathrm{dL}$; WBC $7.34 \times 10^{9} / \mathrm{L}$; platelet $173.0 \times 10^{9} / \mathrm{L}$; CRP $24.93 \mathrm{mg} / \mathrm{L}$; sodium $134 \mathrm{mmol} / \mathrm{L}$; potassium $4.0 \mathrm{mmol} / \mathrm{L}$; chloride $98 \mathrm{mmol} / \mathrm{L}$; urea $8.6 \mathrm{mmol} / \mathrm{L}$; creatinine $133 \mu \mathrm{mol} / \mathrm{L}$; negative dengue NS1 antigen; weakly reactive dengue IgG; negative dengue IgM; bilirubin $7 \mu \mathrm{mol} / \mathrm{L}$; alkaline phosphatase $45 \mathrm{U} / \mathrm{L}$; aspartate transaminase $23 \mathrm{U} / \mathrm{L}$; and alanine transaminase $28 \mathrm{U} / \mathrm{L}$. Nasopharyngeal swab for respiratory panel multiplex by PCR was negative for numerous viruses. Nasopharyngeal swabs for SARS-CoV-2 by PCR were positive on 10 and 11 March. MOH was notified of the COVID-19 case on the evening of 11 March, and a request was made for transfer of care to NCID.

After numerous discussions with NCID and MOH staff on 11-12 March 2020, the instructions from MOH on the afternoon of 12 March were to continue clinical management of Patients A and B at Gleneagles Hospital. A team of private specialists, including respiratory physicians and intensivists, and a cardiologist, nephrologist, haematologist and rheumatologist, was assembled to provide care to the elderly couple.

Patient A continued to have high fever and decreased appetite. She was placed on off-label lopinavir/ritonavir from $13 \mathrm{March}$ 2020. The fever settled from 13 March but recurred on 15 March. Her respiratory symptoms fluctuated in severity. Chest radiography on 16 March revealed bilateral lower zone infiltrates that were more prominent on the left, and she required supplemental intranasal oxygen intermittently from 16 March to 23 March. After initial defervescence, she developed fever again on 20 March. She also developed intolerable gastrointestinal adverse effects from lopinavir/ritonavir, and the decision was made to switch her therapy to a combination of hydroxychloroquine and azithromycin on 20 March. She became afebrile after 20 March. The patient's serial CRP and serum ferritin levels increased, then started to decrease from 28 March (Fig. 1). Serial chest radiographs from 23 March to 2 April demonstrated interval decrease in infiltrates. However, her nasopharyngeal swabs for SARS-CoV-2 by PCR remained persistently positive up to 2 April (Fig. 1). 


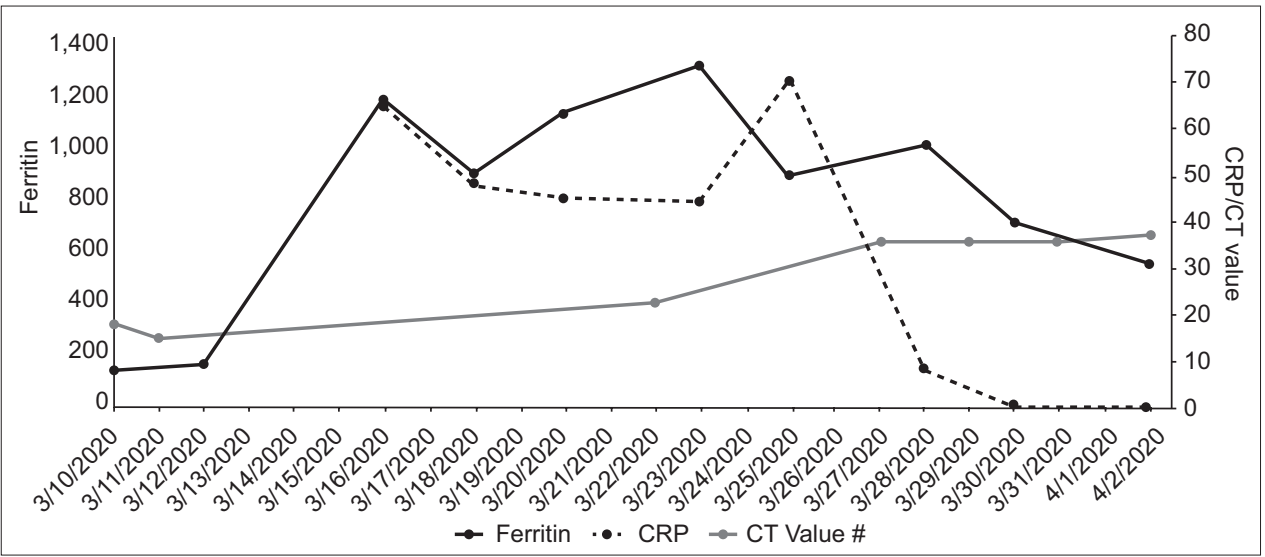

Fig. 1 Graph shows the serial laboratory test results of Patient A from 10 March to 2 April 2020. CRP: C-reactive protein; CT: PCR cycle threshold

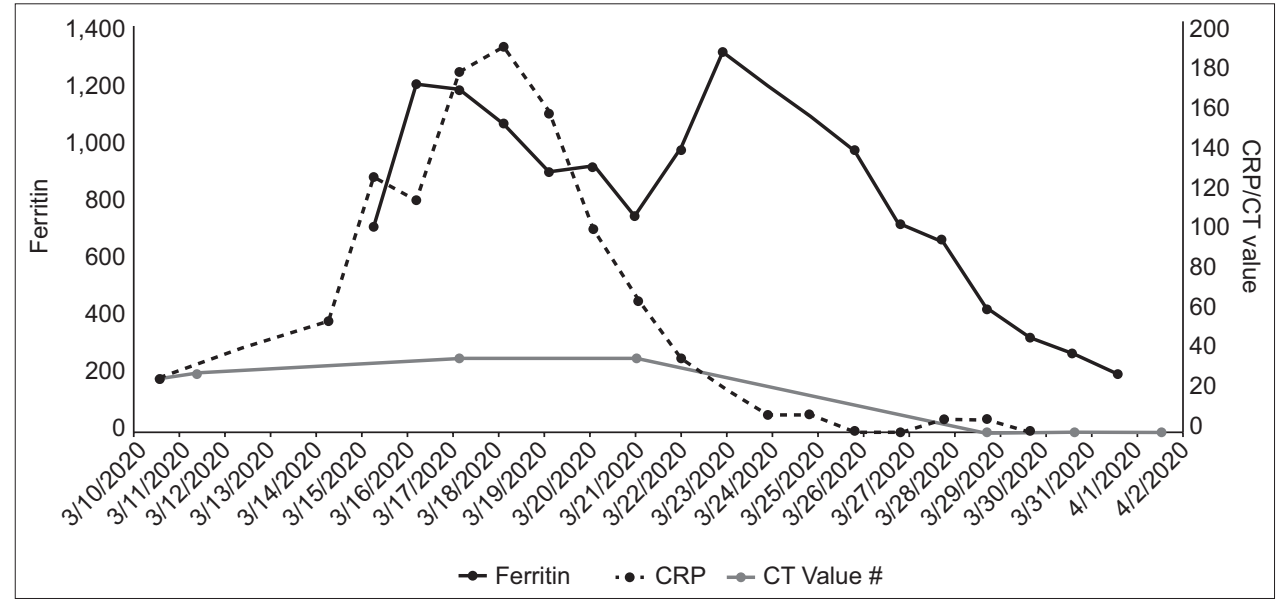

Fig. 2 Graph shows the serial laboratory test results of Patient B from 10 March to 2 April 2020. CRP: C-reactive protein; CT: PCR cycle threshold

Patient A, who had COVID-19 pneumonia, had an estimated case fatality rate of $8 \%$ based on her age of 75 years. ${ }^{(4)}$ She received oral lopinavir/ritonavir, followed by hydroxychloroquine and azithromycin, and recovered well. As of 2 April 2020, she remained hospitalised, as her nasopharyngeal swab was still positive for SARS-CoV-2 by PCR. The role of vaccines to prevent infection and pharmaceutical agents to treat COVID-19 remains under intense study..$^{(5-8)}$

Patient B continued to have high fever and was placed on off-label lopinavir/ritonavir from 12 March 2020. He required supplemental oxygen from 12 March and was monitored in the high dependency unit. As his respiratory status worsened, he was intubated and placed on mechanical ventilation on the early morning of 14 March. He required continuous infusion of noradrenaline to maintain his BP. Baseline electrocardiography showed mild first-degree atrioventricular block and normal QT interval. During his illness, he developed intermittent atrial fibrillation. Echocardiography showed normal resting left ventricular systolic function. He was also placed on IV hydrocortisone $50 \mathrm{mg}$ six-hourly for septic shock. Lopinavir/ritonavir was discontinued and replaced with hydroxychloroquine and azithromycin. QT interval became mildly prolonged, but there was no ventricular arrhythmia. After initial improvement in gas exchange following mechanical ventilation, his ventilatory requirements increased again on 19 March, requiring increases in positive end-expiratory pressure of up to $14 \mathrm{~cm}$ water and fraction of inspired oxygen $\left(\mathrm{FiO}_{2}\right)$ requirements of up to $70 \%$. His temperature failed to lyse despite the change of medication to hydroxychloroquine/azithromycin. In addition, radiological worsening was seen on 20 March. After further discussion, we decided to place him on IV tocilizumab (total dose of 560 mg administered over two days) starting on 20 March. His oxygenation improved, and by 22 March, his $\mathrm{FiO}_{2}$ was reduced to $40 \%$. He became afebrile on 23 March. A percutaneous tracheostomy was performed on 25 March to facilitate weaning from the ventilator, and he was successfully weaned off the ventilator on 31 March. He also developed acute on chronic renal failure with oliguria and was placed on continuous renal replacement therapy from 21 March to 2 April, then subsequently switched to sustained low-efficiency dialysis when he improved haemodynamically. The patient developed progressive thrombocytopenia from 29 March, which was assessed to be drug-related. Heparin and hydroxychloroquine were discontinued on 1 April. Serial laboratory test results are shown in Fig. 2.

Patient B, who developed COVID-19 pneumonia, had several factors that point to a poorer prognosis. He was 82 years of age, had ischaemic heart disease, diabetes mellitus, hypertension and renal impairment, and was an ex-smoker. ${ }^{(9)} \mathrm{A}$ report of 72,314 cases in China documented an overall fatality rate of $2.3 \%$, but that for patients aged above 80 years was $14.8 \%$, and those with ischaemic heart disease $10.5 \%$, diabetes mellitus $9.2 \%$ and hypertension $8.4 \%{ }^{(4)}$ Therefore, it is unsurprising that this patient's illness took a 
rapid deteriorating course - by Day 6 of symptom onset, intubation and mechanical ventilation was required. His clinical condition and chest radiograph findings also worsened, although there was no evidence of new-onset sepsis. The team considered what has been termed a 'cytokine storm' as the underlying reason.

COVID-19 is a coronavirus infection, like the severe acute respiratory syndrome and Middle East respiratory syndrome. In all three infections, there have been well-described abnormalities in the patients involving both the innate and adaptive parts of the immune system. ${ }^{(10)}$ In some patients, pro-inflammatory cytokines such as interleukin 1 (IL-1) and interleukin 6 (IL-6) are upregulated, which leads to further worsening of the lung inflammation and acute respiratory distress syndrome (ARDS). In such instances, anti-cytokine therapy such as anti-IL-6 (e.g. tocilizumab) have been used. Some questions will need to be answered. Who will benefit the most from anti-cytokine therapy? What is the optimal timing to start this therapy given that there is likely to be a window of opportunity for the best outcome? What therapeutic agent should we use? Currently, trials are in progress to determine the answers to these questions.

Not all patients will require anti-cytokine therapy. In the Chinese experience, levels of CRP and serum IL-6, and lung ultrasonography have been used to guide therapy. In the absence of more definitive data, the first signal may be what is known as a 'double dip' phenomenon in the patient's clinical progress. Patient B initially responded to mechanical ventilation but deteriorated again (the second dip) four days later. At that time, it was essential to rule out other reasons for his deterioration. Some of these reasons included a superimposed new infection or myocardial injury, which has been described in patients with COVID-19 infections ${ }^{(11,12)}$ It is important $^{\text {in }}$ to start immunomodulation before a patient develops persistent ARDS and lung damage. Ferro et al, in a recent editorial, postulated that the window of opportunity to use anti-cytokine therapy is between Day 7 and Day 14 of illness in patients who develop worsening lung disease. ${ }^{(13)}$ There is also uncertainty about the best therapeutic agent to use. In such patients, pro-inflammatory cytokines IL-1 and IL-6 play a prominent role. Anti-IL-1 agents such as anakinra have more limited usage compared to anti-IL-6 agents such as tocilizumab, which is approved for treatment of rheumatoid arthritis and the cytokine storm invoked by immune treatment with chimeric antigen receptor T cells in cancer patients. We were fortunate that Patient B appeared to be recovering well. We started tocilizumab before his ARDS worsened and eight days later, he had improved enough to be weaned off ventilatory support.

While we agree that the way forward in the management of COVID-19 is to enrol patients in carefully designed clinical trials, ${ }^{(14)}$ we are also in the midst of an ongoing outbreak with significant mortality in the elderly. In the absence of definitive results from such trials, our experience using a risk-adapted treatment approach in the clinical management of this elderly couple with a markedly different course of illness may shed light on the management of elderly patients diagnosed with COVID-19 infection.

Yours sincerely,

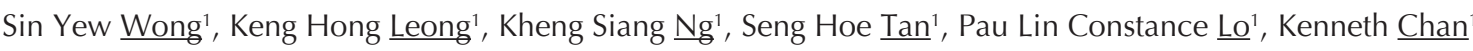

${ }^{1}$ Gleneagles Medical Centre, Singapore. symswong@singnet.com.sg

\section{References}

1. Young BE, Ong SWX, Kalimuddin S, et al. Epidemiologic features and clinical course of patients infected with SARS-CoV-2 in Singapore. JAMA 2020; 323:1488-94.

2. $\mathrm{Ng} \mathrm{Y,} \mathrm{Li} \mathrm{Z,} \mathrm{Chua} \mathrm{YX,} \mathrm{et} \mathrm{al.} \mathrm{Evaluation} \mathrm{of} \mathrm{the} \mathrm{effectiveness} \mathrm{of} \mathrm{surveillance} \mathrm{and} \mathrm{containment} \mathrm{measures} \mathrm{for} \mathrm{the} \mathrm{first} 100$ patients with COVID-19 in Singapore - January 2-February 29, 2020. MMWR Morb Mortal Wkly Rep 2020; 69:307-11.

3. Hsu LY, Chia PY, Vasoo S. A midpoint perspective on the COVID-19 pandemic. Singapore Med J 2020; 61:381-3.

4. Wu Z, McGoogan JM. Characteristics of and important lessons from the coronavirus disease 2019 (COVID-19) outbreak in China: summary of a report of 72314 cases from the Chinese Center for Disease Control and Prevention. JAMA 2020 Feb 24. https://doi.org/10.1001/jama.2020.2648. [Epub ahead of print]

5. Pang J, Wang MX, Ang IYH, et al. Potential rapid diagnostics, vaccine and therapeutics for 2019 novel coronavirus (2019-nCoV): a systematic review. J Clin Med 2020; 9. pii:E623.

6. Lai CC, Liu YH, Wang CY, et al. Asymptomatic carrier state, acute respiratory disease, and pneumonia due to severe acute respiratory syndrome coronavirus 2 (SARSCoV-2): facts and myths. J Microbiol Immunol Infect 2020; 53:404-12.

7. Devaux CA, Rolain JM, Colson P, Raoult D. New insights on the antiviral effects of chloroquine against coronavirus: what to expect for COVID-19? Int J Antimicrob Agents 2020; 55:105938.

8. Cao B, Wang Y, Wen D, et al. A trial of lopinavir-ritonavir in adults hospitalized with severe Covid-19. N Engl J Med 2020; 382:1787-99.

9. Wang L, He W, Yu X, et al. Coronavirus Disease 2019 in elderly patients: characteristics and prognostic factors based on 4-week follow-up. J Infect 2020; 80:639-45.

10. Sarzi-Puttini P, Giorgi V, Sirotti S, et al. COVID-19, cytokines and immunosuppression: what can we learn from severe acute respiratory syndrome? Clin Exp Rheumatol 2020; 38:337-42.

11. Shi S, Qin M, Shen B, et al. Association of cardiac injury with mortality in hospitalized patients with COVID-19 in Wuhan, China. JAMA Cardiol 2020 Mar 25. https:// doi.org/10.1001/jamacardio.2020.0950. [Epub ahead of print]

12. Cherian R, Poh KK. At the 'heart' of the COVID-19 outbreak: early cardiac implications and mitigating strategies. Singapore Med J 2020; 61:373-4.

13. Ferro F, Elefante E, Baldini C, et al. COVID-19: the new challenge for rheumatologists. Clin Exp Rheumatol 2020; 38:175-80.

14. Kalil A. Treating COVID-19-off-label drug use, compassionate use and randomized clinical trials during pandemics. JAMA 2020 Mar 24. https://doi.org/10.1001/ jama.2020.4742. [Epub ahead of print] 